\title{
Towards quantitative, atomic-resolution reconstruction of the electrostatic potential via differential phase contrast using electrons
}

\author{
R. Close ${ }^{\mathrm{a}}$, Z. Chen ${ }^{\mathrm{a}}$, N. Shibata ${ }^{\mathrm{b}}$, S.D. Findlay ${ }^{\mathrm{a}, *}$ \\ ${ }^{a}$ School of Physics and Astronomy, Monash University, Clayton, Victoria 3800, Australia \\ ${ }^{b}$ Institute of Engineering Innovation, School of Engineering, University of Tokyo, Tokyo 113-8656, Japan
}

\begin{abstract}
Differential phase contrast images in scanning transmission electron microscopy can be directly and quantitatively related to the gradient of the projected specimen potential provided that (a) the specimen can be treated as a phase object and (b) full 2D diffraction patterns as a function of probe position can be obtained. Both are challenging to achieve in atomic resolution imaging. The former is fundamentally limited by probe spreading and dynamical electron scattering, and we explore its validity domain in the context of atomic resolution differential phase contrast imaging. The latter, for which proof-of-principle experimental data sets exist, is not yet routine. We explore the extent to which more established segmented detector geometries can instead be used to reconstruct a quantitatively good approximation to the projected specimen potential.
\end{abstract}

Keywords: Scanning transmission electron microscopy (STEM); Differential phase contrast (DPC) imaging

\section{Introduction}

Early work by Rose [1, 2] and Dekkers and de Lang [3] shows that taking the difference between the scanning transmission electron microscopy (STEM) images from suitably configured detector segments produces images with contrast relating closely to the phase profile imparted on the electron beam by the electrostatic potential of the specimen. For a configuration involving quadrant detectors, the standard conceptual picture is that the difference signal between diametrically opposed detector segments is proportional to the beam deflection along the direction between the segments, which in turn is proportional to the gradient of the phase profile in that direction. This imaging mode is therefore called differential phase contrast (DPC). This technique has been used to great effect to image magnetic domain structure in materials [4-8], and more recently to image electric fields [9-12]. In all these cases, the lateral extent of the probe is significantly smaller than that of the variations in the magnetic or electric fields of interest - a circumstance that considerably simplifies the analysis and interpretation [13]. However, Shibata et al. [10] and Müller et al. [14] have recently demonstrated that DPC imaging can be accomplished at atomic resolution, where the probe size is larger than the scale on which the atomic potentials vary.

Figure 1(a) shows a high-angle annular dark field (HAADF) image for a thin specimen $(\sim 30 \AA)$ of $\mathrm{SrTiO}_{3}$ viewed along the [001] zone axis, with $200 \mathrm{keV}$ electrons and a probe-forming aperture semiangle of $23 \mathrm{mrad}$. The atomic columns appear as bright peaks, with intensities roughly proportional to the square of the atomic number $Z$ of the elements in the column, giving robustly interpretable $Z$-contrast imaging [15]. Figure 1(b)

${ }^{*}$ Corresponding author: scott.findlay@monash.edu shows the DPC image for quadrant detector segments aligned along the horizontal direction in the figure and spanning the scattering angle range 15.3-30.7 mrad. Each atomic column in Fig. 1(b) appears as a bow-tie pattern with one dark (negative) lobe and one light (positive) lobe. This is qualitatively consistent with the derivative in the horizontal direction of the projected electrostatic potential of the structure. ${ }^{1}$

A broad conceptual understanding of the appearance of the DPC image can be developed as follows. Because the fast electron penetrates the atomic electron clouds, the net force it experiences tends to be attraction to the not-fully-screened nuclei. Thus, as sketched in Fig. 1(c), when a fine electron probe passes to the left of a column of atoms it is deflected to the right by the attractive electric field of the column, increasing the signal on the right hand detector segment while reducing it on the left hand detector segment. The reverse happens when the probe passes to the right of the column. The contrast in the DPC image, which is formed from the difference between the individual signals from each detector as a function of probe position, thus reverses as the probe is scanned across an atomic column, as seen in Fig. 1(b). As recently emphasised by Lubk and Zweck [13] and Müller et al. [14], the "cartoon" depiction of Fig. 1(c) is rather simplistic. The reality is more like what is sketched in Fig. 1(d): due to electron scattering in the specimen, the intensity distribution in the diffraction pattern is rather more complicated than a simple rigid translation of the bright field disk. Moreover, strongly-thickness-dependent electron multiple scattering and coherent interference effects in the bright field region make atomic resolution DPC imaging much less robust than

\footnotetext{
${ }^{1}$ This may be roughly gauged from the HAADF image in Fig. 1(a), which is qualitatively reminiscent of the specimen potential, save that DPC signal is also evident for the pure oxygen columns invisible in the HAADF image.
} 


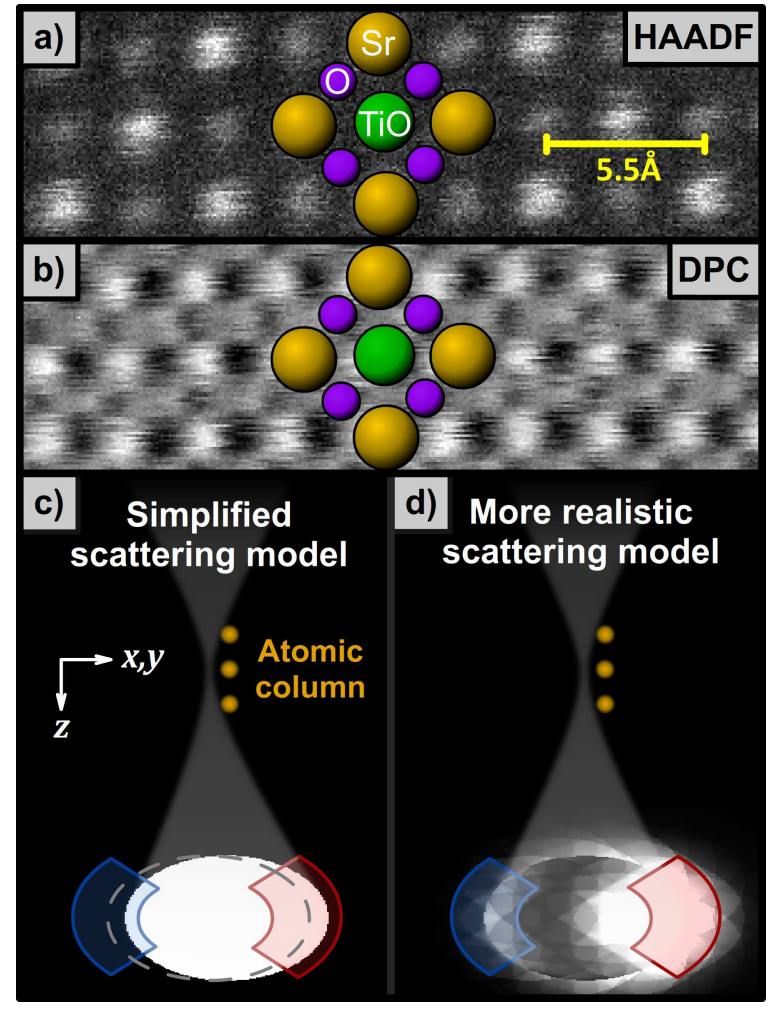

Figure 1: Simultaneously acquired experimental (a) HAADF and (b) DPC images of $\mathrm{SrTiO}_{3}$ for a $30 \AA$ thick specimen oriented along the [001] zone axis (adapted from data in Ref. [10]). (c) Idealized DPC schematic where the STEM probe deflection results in a simple translation (from the dashed line reference) of the bright field disk across the diffraction plane and the two detector segments shown. (d) More realistic DPC schematic where the interaction of the STEM probe with the column of atoms still leads to a net deflection but the intensity redistribution in the diffraction plane is more complex.

HAADF. This is seen in Fig. 2 which shows defocus-thickness tableaux of HAADF and DPC images for $\mathrm{SrTiO}_{3}$. Whereas the appearance of the HAADF images are largely unchanged over a wide range of thickness and defocus values, the DPC images are much more sensitive to these parameters.

The moniker "differential phase contrast" implies that the images bear close resemblance to the gradient of the phase $[2,3,16,17]$. However, the majority of proposals for reconstructing the specimen potential in such cases assume the specimen to be a weak phase object, and as such are not fundamentally different from other analysis / reconstruction approaches making the same assumption (e.g. Refs. [1, 18-24]). In particular, Pennycook et al. have recently reconstructed the potential of a bilayer of graphene using a pixel detector [23]. However, in atomic resolution electron microscopy, the validity domain of the weak-phase-object approximation is extremely limited. It breaks down in the presence of strong scattering $[25,26]$ and through its neglect of the spatial propagation of the wavefunction [26-28], especially in the high-resolution regime [26, 29]. DPC imaging can to some extent overcome the strong scattering limitation: it applies equally for strong phase objects provided either that the phase gradients are constant over the size of the probe $[13,30,31]$ or else that the displacement of the "cen-

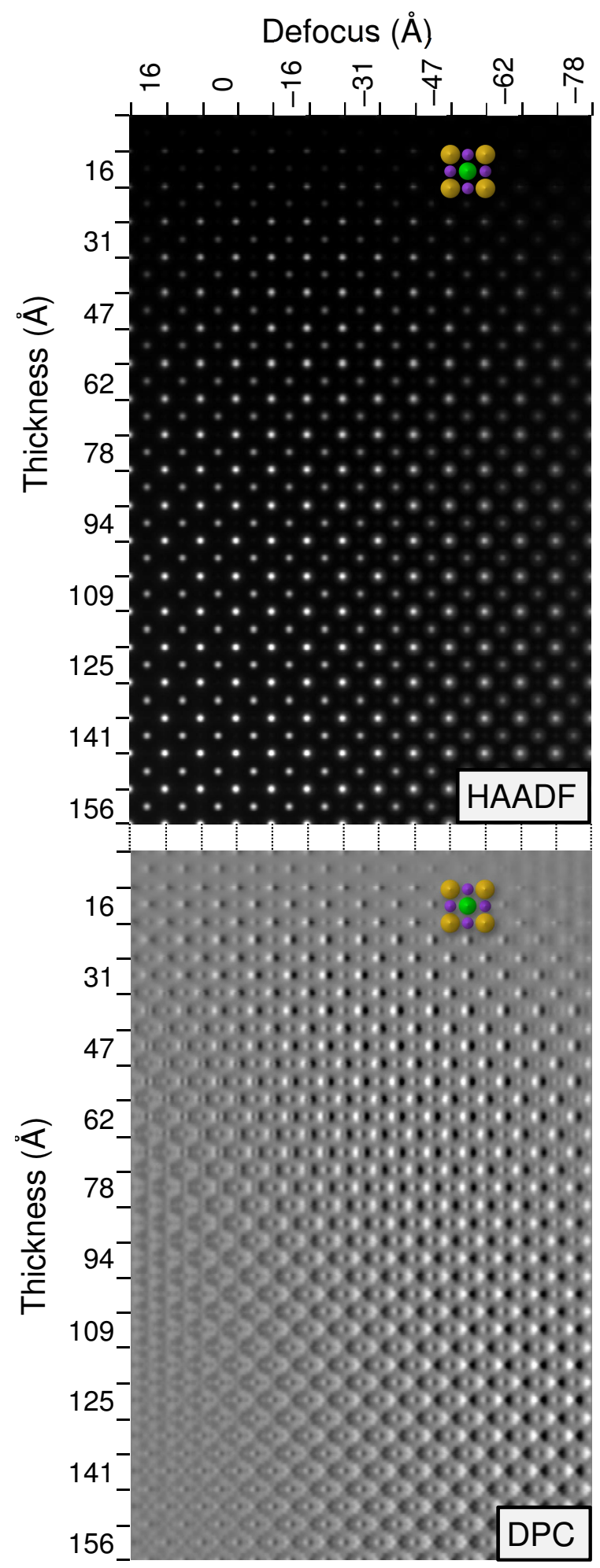

Figure 2: Defocus-thickness tableaux for $\mathrm{HAADF}$ and DPC imaging of $\mathrm{SrTiO}_{3}$ oriented along the [001] zone axis, assuming $200 \mathrm{keV}$ electrons and a probeforming aperture angle of $23 \mathrm{mrad}$. The HAADF detector spans the range 81228 mrad. The DPC signal is based on diametrically opposite quadrants in a ring spanning 15.3-30.7 mrad. Positive defocus values correspond to overfocus. 
tre of mass", i.e. the first moment, of the diffraction pattern intensity distribution can reliably be determined $[13,14,30]$. The neglect-of-propagation limitation, though, is at present surmountable only through comparison with detailed simulations.

In this paper we explore the validity domain of the phaseobject approximation when seeking to quantitatively analyse DPC STEM imaging at atomic resolution to reconstruct the (projected) specimen potential, from which details on atom location, atom species, and possibly bonding, might be deduced. We will consider both pixel detectors $[14,23,32]$ and the extent to which such imaging can be approximately realized with the more established segmented detector set-up $[9,33,34]$.

\section{An overview of phase retrieval approaches}

The multislice method simulates a fast electron probe scattering through a crystal via an alternating sequence of phase grating and propagation steps, the former describing scattering from the specimen potential and the latter spatial propagation forward through the crystal [35]. The so-called multiplicativeobject approximation consists of neglecting all the propagation steps. The wavefunction at the exit surface may then be written as the product of the wavefunction at the entrance surface and a single phase grating or transmission function:

$$
\psi_{\text {exit }}\left(\mathbf{r}_{\perp}\right)=\psi_{\text {entrance }}\left(\mathbf{r}_{\perp}\right) q\left(\mathbf{r}_{\perp}\right),
$$

where

$$
q\left(\mathbf{r}_{\perp}\right)=\exp \left\{i \sigma\left[V\left(\mathbf{r}_{\perp}\right)+i V^{\prime}\left(\mathbf{r}_{\perp}\right)\right] t\right\},
$$

in which $\mathbf{r}_{\perp}$ is the coordinate in the plane of the specimen, $V\left(\mathbf{r}_{\perp}\right)$ is the projected (and thermally-smeared) elastic potential, $V^{\prime}\left(\mathbf{r}_{\perp}\right)$ is the absorptive potential (due primarily to thermal scattering), $\sigma=2 \pi m e \lambda / h^{2}$ is an electron-energy-dependent interaction constant [35] (both $m$ and $\lambda$ incorporating relativistic corrections), and $t$ denotes the sample thickness.

The potential for absorption is significantly smaller than that for elastic scattering, and thus the transmission function mainly modifies the phase of the fast electron wavefunction. ${ }^{2}$ Moreover, this phase is proportional to the projected specimen potential, which contains atom location, species, and bonding information. However, phase is not directly accessible - experiments only measure intensities. Much research in electron microscopy has thus been concerned with strategies to obtain intensity contrast related to the exit surface phase, or, more quantitatively, to reconstruct that phase from intensity measurements.

We cannot here undertake the formidible task of overviewing all the strategies for phase retrieval. However, the wider context helps to understand the strengths and weaknesses of STEM DPC, so a simple summary of some of the main atomicresolution phase retrieval methods will be attempted. It is useful to distinguish different levels of approximation: techniques invoke the weak-phase-object approximation if they linearize the

\footnotetext{
${ }^{2}$ The terms "multiplicative-object approximation", "phase-object approximation" and "projection approximation" are thus often used more-or-less interchangeably in the literature [26].
}

relation between measured quantities and the specimen potential; techniques invoke the strong-phase-object approximation if they retain the nonlinear relation to the specimen potential implied by Eq. (2).

In conventional transmission electron microscopy (CTEM), "phase retrieval" has a direct interpretation: one seeks to determine the phase of the exit surface wavefunction which, if the phase object approximation holds, is precisely the transmission function of Eq. (2) because the incident wave has uniform intensity and phase over a wide area. Strategies for CTEM phase retrieval include through-focal series [36-41], tilt series $[42,43]$, coherent diffractive imaging [44-47], and holography $[48,49]$.

Methods reconstructing either the phase or projected potential in Eq. (2) after processing STEM data can also be considered forms of phase retrieval, even though the convergent probe only samples the transmission function of Eq. (2) in a sequence of patches as the probe is scanned across the specimen and the full transmission function is thus not the phase of any single STEM wavefunction. This sequential acquisition introduces scan distortions to which CTEM imaging is immune. However, the strength of STEM is the ease with which multiple imaging modes can be acquired simultaneously, some of which offer robustly interpretable imaging [15] that can inform or constrain other analyses.

Within the weak-phase-object approximation, the specimen potential can in principle be determined from the signal in a single STEM detector $[1,21]$. However, reconstructions tend to be improved by combining results from multiple detectors [2, 18-21], a variant on ptychography. A recent weak-phaseobject analysis using a pixel detector [23] shows that it offers greater flexibility for optimising signal-to-noise [24].

In the strong-phase-object approximation, pixel detectors enable ptychographic reconstructions via either the Wignerdistribution deconvolution approach [50] or iterative strategies for coherent diffractive imaging [51-53]. The latter has been realized in atomic resolution STEM [54-56]; the former, so far as we are aware, has not. STEM DPC can quantitatively effect phase retrieval of strong phase objects since the displacement of the first moment of the diffraction pattern is closely related to the gradient of the phase [13, 14, 30].

The different approaches can usefully be compared on the following criteria:

Resolution: Excepting coherent diffractive imaging, ${ }^{3}$ CTEM techniques are diffraction limited by the image-forming lens aperture (or incoherent aberrations, which impose a similar limit). Both weak-phase-object and strong-phase-object STEM DPC analysis is diffraction limited, but by twice the probe-forming aperture (the transfer function being the Fourier transform of the probe intensity rather than the probe amplitude), offering a factor of two resolution improvement over most CTEM approaches. Neither coherent diffractive imaging CTEM nor STEM ptychography are diffraction limited in principle $[53,57]$, though in practice signal-to-noise ultimately imposes a limit on achievable resolution.

\footnotetext{
${ }^{3}$ And arguably tilt series, which can extend the diffraction limit [43].
} 
Computational complexity: Off-axis holography, weakphase-object approaches, Wigner-distribution deconvolution ptychography and STEM DPC all offer deterministic reconstruction procedures, which tend to be computationally efficient. The other CTEM approachs and iterative approaches to STEM ptychography do not need to invoke the weak-phaseobject approximation, but the analysis then relies on methods to handle the nonlinear relation between the measured intensities and the sought phase, which tends to require substantial post-acquisition processing and raises questions of uniqueness.

Domain of validity: The strong-phase-object approximation has wider validity than the weak-phase-object approximation, but both break down for sufficiently strongly scattering specimens, as governed by composition and thickness [25-29]. The point of breakdown and its consequences for phase retrieval may, however, depend on the imaging mode and analysis method. The present paper explores this question for STEM DPC. Excepting tilt series, CTEM methods have greater validity than the phase object approximation - they can reconstruct the exit surface wavefunction regardless of the degree of multiple scattering, though quantitative interpretation in this regime would require the inversion of the multiple scattering. Iterative STEM ptychography offers similar prospects for overcoming multiple scattering [58], though they have not yet been realised at atomic resolution.

Returning to the focus of the present paper, atomic-resolution STEM DPC offers a deterministic procedure based on analysing the first moment of the diffraction pattern. It is diffraction limited, but by a transfer function given by the probe intensity, which is not overly restrictive in aberration-corrected STEM. However, as we will show, the validity domain of the strongphase-object approximation on which STEM DPC analysis relies is quite restrictive at atomic resolution. Realised using a pixel detector, the data set would also be suitable for ptychographic analysis, which could, at the expense of the greater computational effort, improve the signal-to-noise [24] and extend the diffraction limit. It may approximately be realised using segmented detectors, which give a coarser estimate for the first moment of the diffraction pattern [13]. In principle, pixel detectors are thus always superior for precision work. That said, segmented detector STEM DPC has the advantages of being easy to implement with established detector technology, involving much smaller data sets, and allowing sufficently rapid processing as to enable so-called "live imaging" [12]. Since, as we shall show, the approximation segmented detectors provide for the first moment can be quite good, the advantages of this approach are not inconsiderable.

\section{Theoretical framework}

This section summarizes the key steps in the theoretical analysis. The reader is referred to the literature for greater detail $[2,13,14,16,30,59]$.

We adopt a coordinate system where the optical axis is the $z$-axis, $\mathbf{r}_{\perp}$ denotes vectors in the plane perpendicular to this, $\mathbf{k}_{\perp}$ denotes the Fourier space coordinate conjugate to $\mathbf{r}_{\perp}$, and $\mathbf{R}$ denotes the position of the STEM probe on the specimen surface. The wavefunction of the electron probe at the specimen entrance surface may then be written

$$
t\left(\mathbf{r}_{\perp}-\mathbf{R}\right)=\int T\left(\mathbf{k}_{\perp}\right) e^{2 \pi i \mathbf{k}_{\perp} \cdot\left(\mathbf{r}_{\perp}-\mathbf{R}\right)} d \mathbf{r}_{\perp},
$$

where $T\left(\mathbf{k}_{\perp}\right)=O\left(k_{\perp}\right) \exp \left[-2 \pi i \chi\left(\mathbf{k}_{\perp}\right) / \lambda\right]$ is the lens transfer function in which $O\left(k_{\perp}\right)$ is the objective pupil aperture function and $\chi\left(\mathbf{k}_{\perp}\right)$ is the objective aperture aberration function.

Using Eq. (3) as the entrance surface wavefunction in Eq. (1), the intensity in the diffraction pattern can be shown to be

$$
I\left(\mathbf{k}_{\perp}, \mathbf{R}\right)=\left|\int T\left(\mathbf{k}_{\perp}-\mathbf{k}_{\perp}^{\prime}\right) Q\left(\mathbf{k}_{\perp}^{\prime}\right) e^{2 \pi i \mathbf{k}_{\perp}^{\prime} \cdot \mathbf{R}} d \mathbf{k}_{\perp}^{\prime}\right|^{2},
$$

where $Q\left(\mathbf{k}_{\perp}\right)$ is the Fourier transform of $q\left(\mathbf{r}_{\perp}\right)$. If, rather than obtaining full diffraction patterns, one uses an integrating detector giving the total intensity within an extended area in the diffraction plane, the resultant STEM image may be written

$$
I_{j}(\mathbf{R})=\int I\left(\mathbf{k}_{\perp}, \mathbf{R}\right) D_{j}\left(\mathbf{k}_{\perp}\right) d \mathbf{k}_{\perp},
$$

where $D_{j}\left(\mathbf{k}_{\perp}\right)$ is the response function for detector segment $j$, the index allowing that images from more than one detector segment may be recorded simultaneously.

The primary limitation on determining the specimen structure from experimental measurements of $I\left(\mathbf{k}_{\perp}, \mathbf{R}\right)$ is that Eq. (4) depends nonlinearly on the transmission function. The weakphase-object approximation resolves this via linearization: it assumes that

$$
q\left(\mathbf{r}_{\perp}\right)=1+\tilde{q}\left(\mathbf{r}_{\perp}\right)
$$

and that terms quadratic in $\tilde{q}$ may be neglected. ${ }^{4}$ Within this approximation, the Fourier transform of Eq. (4) with respect to probe position may then be written as

$$
\begin{aligned}
I\left(\mathbf{k}_{\perp}, \mathbf{K}\right)= & \left|T\left(\mathbf{k}_{\perp}\right)\right|^{2} \delta(\mathbf{K}) \\
& +i \tilde{Q}_{i}(\mathbf{K})\left[T^{*}\left(\mathbf{k}_{\perp}\right) T\left(\mathbf{k}_{\perp}-\mathbf{K}\right)-T\left(\mathbf{k}_{\perp}\right) T^{*}\left(\mathbf{k}_{\perp}+\mathbf{K}\right)\right] \\
& +\tilde{Q}_{r}(\mathbf{K})\left[T^{*}\left(\mathbf{k}_{\perp}\right) T\left(\mathbf{k}_{\perp}-\mathbf{K}\right)+T\left(\mathbf{k}_{\perp}\right) T^{*}\left(\mathbf{k}_{\perp}+\mathbf{K}\right)\right] .
\end{aligned}
$$

where $\tilde{Q}_{r}$ and $\tilde{Q}_{i}$ are the Fourier transforms of the real and imaginary components of $\tilde{q}$, respectively. The result is a set of linear equations from which $\tilde{Q}_{r}(\mathbf{K})$ and $\tilde{Q}_{i}(\mathbf{K})$ can be determined from the set of non-zero values of $I\left(\mathbf{k}_{\perp}, \mathbf{K}\right)$. This structure is preserved in the case of integrating detectors.

However, Waddell and Chapman [30] proposed a different approach to getting around the non-linearity of Eq. (4). They consider a so-called "first-moment detector" which can be used to determine the centre of the intensity distribution in two perpendicular directions. As recently demonstrated by Müller [14], this can be achieved from a pixel detector by synthesizing

\footnotetext{
${ }^{4}$ Practice varies as to whether Eq. (6) is taken to be the Taylor expansion of Eq. (2) to first order in $\sigma$, such that neglecting quadratic terms in $\tilde{q}$ is selfconsistent, or whether Eq. (6) is exact and neglecting quadratic terms in $\tilde{q}$ is simply a convenient assumption. We suspect that when the approximation holds, the difference between these approaches is negligible.
} 
images assuming $D_{x}\left(\mathbf{k}_{\perp}\right)=k_{x}$ and $D_{y}\left(\mathbf{k}_{\perp}\right)=k_{y}$. Considering just the $x$-direction (the $y$-direction form follows trivially) and assuming a rotationally symmetric probe, ${ }^{5}$ it can be shown that

$$
\begin{aligned}
I_{x}(\mathbf{R}) & \equiv \int I\left(\mathbf{k}_{\perp}, \mathbf{R}\right) k_{x} d \mathbf{k}_{\perp} \\
& =\frac{1}{2 \pi}\left[A_{q}^{2}(\mathbf{R}) \frac{\partial}{\partial R_{x}} \phi_{q}(\mathbf{R})\right] \otimes|t(\mathbf{R})|^{2},
\end{aligned}
$$

where $\otimes$ denotes convolution and $A_{q}$ and $\phi_{q}$ are the amplitude and phase of $q$, i.e.

$$
q\left(\mathbf{r}_{\perp}\right)=A_{q}\left(\mathbf{r}_{\perp}\right) \exp \left[i \phi_{q}\left(\mathbf{r}_{\perp}\right)\right] .
$$

Neglecting the amplitude contrast, since the cross-section for inelastic scattering is weaker than that for elastic scattering and the samples we will consider are thin, and recognising that the differential operator then commutes with the convolution, such a detector realizes exact differential contrast of the form: ${ }^{6}$

$$
I_{x}(\mathbf{R})=\frac{1}{2 \pi} \frac{\partial}{\partial R_{x}}\left[\phi_{q}(\mathbf{R}) \otimes|t(\mathbf{R})|^{2}\right] .
$$

One approach to solving this equation which is particularly convenient for periodic crystals is the Fourier derivative theorem [31], which gives that

$$
\phi_{q}(\mathbf{R}) \otimes|t(\mathbf{R})|^{2}=\mathcal{F}^{-1}\left\{\frac{\mathcal{F}\left[I_{x}(\mathbf{R})\right]+i \mathcal{F}\left[I_{y}(\mathbf{R})\right]}{i\left(k_{x}+i k_{y}\right)}\right\},
$$

where $\mathcal{F}$ denotes Fourier transformation between coordinates $\mathbf{R}$ and $\mathbf{k}_{\perp}$. The divergence of the denominator in Eq. (12) is handled by setting the term in braces equal to zero at $\mathbf{k}_{\perp}=\mathbf{0}$, which means that we can only reconstruct phase differences and not the absolute phase. Deconvolving the probe intensity from Eq. (12) by Fourier methods enables us to conveniently express the result in the same framework:

$$
\phi_{q}(\mathbf{R})=\mathcal{F}^{-1}\left\{\frac{\mathcal{F}\left[I_{x}(\mathbf{R})\right]+i \mathcal{F}\left[I_{y}(\mathbf{R})\right]}{i\left(k_{x}+i k_{y}\right) \mathcal{F}\left[|t(\mathbf{R})|^{2}\right]}\right\} .
$$

Since the probe intensity is bandwidth limited, we must now zero the expression in braces for all $\left|\mathbf{k}_{\perp}\right|$ beyond twice the reciprocal-space probe-forming aperture cut-off, since it is $a$ priori known that $\mathcal{F}\left[|t(\mathbf{R})|^{2}\right]=0$ in that region. Consequently, the higher spatial frequencies of the phase and thus the projected specimen potential, which broadly correspond to potential variations on scales notably smaller than the size of the probe, cannot be reconstructed via this approach. Figure 3

\footnotetext{
${ }^{5}$ This assumption is not strictly necessary, but eliminates the need for a second term accounting for a contribution to the signal from differential absorption contrast and/or that the centre of the intensity distribution in the absence of a specimen might not coincide with the chosen origin for $\mathbf{k}_{\perp}$ space [30].

${ }^{6}$ Lubk and Zweck [13] and Müller et al. [14] present alternative derivations which interpret Eq. (8) as being proportional to the expectation value of the momentum transfer, which they elegantly relate to the projection of the electromagnetic field inside the specimen. However, we will persist in seeking to determine the phase of the transmission function.
}

shows how the specimen potentials for $\mathrm{SrTiO}_{3}$ and $\mathrm{Al}_{3} \mathrm{Li}$ are affected by the bandwidth limiting appropriate to the experimental conditions of the data shown in Fig. 1(a,b). The effect is non-negligible, though does not prevent identification of the atom locations or discrimination between different atomic species. Indeed, since the high frequency potential components are dominated by the nuclear contribution, they are arguably of less interest than the low frequency potential components which contain more information about the electron density. It should be emphasized that this bandwidth limiting has no impact on the values of those lower frequency components within the bandwidth limit - they, up to the validity of the phase object approximation and other limiting experimental effects discussed later, remain fully reconstructable.
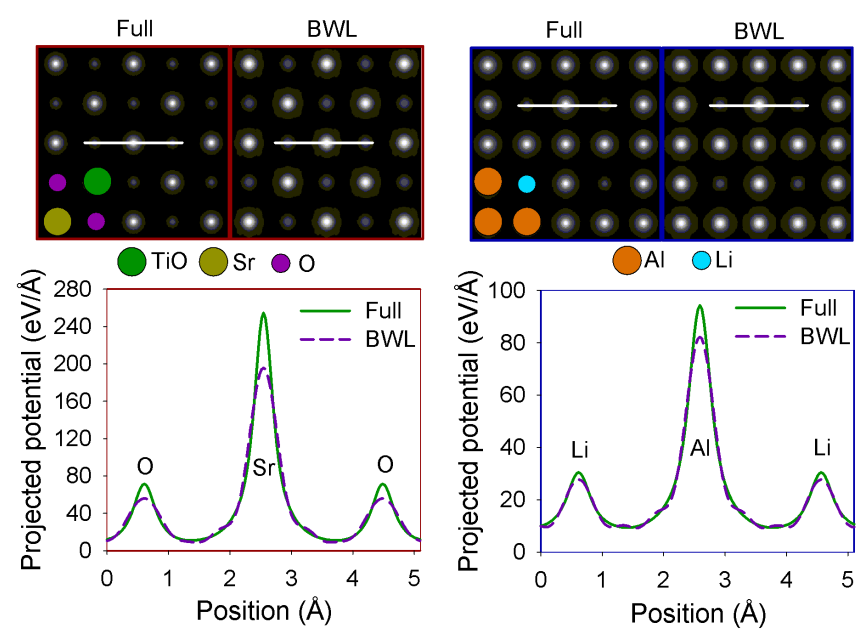

Figure 3: Projected specimen potentials for $\mathrm{SrTiO}_{3}$ [001] (left) and $\mathrm{Al}_{3} \mathrm{Li}$ [001] (right), comparing the "full" potential, i.e. including all frequencies, against the bandwidth limited "BWL" potential, which includes only spatial frequencies up to $1.8 \AA^{-1}$, as appropriate to the information irretrievably lost to DPC imaging via the convolution in Eq. (12). A $200 \mathrm{keV}$ probe with $23 \mathrm{mrad}$ probe-forming aperture semiangle has been assumed. In later figures, linescan extracts average over a finite width as per the standard experimental approach to noisy data. Since that approach gives almost indistinguishable profiles if used here, instead the linescans shown are only a single pixel wide to highlight the quantitative error in the peaks due to the removal the high spatial frequency components.

In the presence of noise, it may further be necessary to suppress those frequencies for which $\mathcal{F}\left[|t(\mathbf{R})|^{2}\right]$ is small, say via Tikhonov regularization [60]. Note, though, that the integration already goes some way towards suppressing high-frequency noise via the increasing magnitude of the $k_{x}+i k_{y}$ term in the denominator.

In summary, when the first-moment detector DPC signal can be synthesized [14], Eq. (13) provides a prescription to reconstruct $\phi_{q}(\mathbf{R})$, up to the bandwidth limit imposed by the STEM image formation process.

\section{Scattering and propagation as fundamental limits on phase reconstruction with a first-moment detector}

Since Eqs. (9) and (13) are based on the phase-object approximation, the validity domain of the first-moment detector 
DPC phase reconstruction is expected to be wider than that of the weak-phase-object approximation phase reconstruction, Eq. (6), i.e. it should hold for thicker and/or more strongly scattering specimens. Nevertheless, the multiplicative form of Eq. (1) is an approximation. We explore this fundamental limitation by simulating multiple elastic scattering using the multislice method when calculating the STEM DPC signal of Eq. (8), and then endeavouring to reconstruct the phase via Eq. (13). Comparison of the reconstruction with the ideal phase-object approximation phase (from Eq. (2)) will establish the quantitative fidelity of the reconstruction as a function of thickness.

Our exploration is based on parameters appropriate to the experimental data in Figs. 1(a) and (b): $200 \mathrm{keV}$ electrons and a probe-forming aperture semiangle of $23 \mathrm{mrad}$, typical values for atomic resolution imaging. We consider both $\mathrm{SrTiO}_{3}$ and $\mathrm{Al}_{3} \mathrm{Li}$ as test samples, the former a strongly scattering specimen and the latter, comprising only light weight elements, a weakly scattering specimen. Consistent with the improved phase-object approximation approach [61, 62], the defocus is set to nominally (i.e. in the absence of scattering) place the beam waist in the midplane of the crystal. Inspection of the DPC tableau in Fig. 2 shows that the qualitative appearance of the DPC images conforms to this prescription for the optimum defocus. Section 6 explores the error introduced if this condition is not achieved.

Two error metrics are considered for comparisons between the reconstructed phase of Eq. (13) and the ideal phase of Eq. (2). The first metric is a mean square error:

$$
\text { MSE }=\frac{1}{N_{\text {pixels }}} \frac{\sum_{\text {all pixels } i}\left|\phi_{q}\left(\mathbf{R}_{i}\right)-\sigma \widetilde{V}\left(\mathbf{R}_{i}\right) t\right|^{2}}{t^{2}} .
$$

The potential $\widetilde{V}(\mathbf{R})$ is a filtered version of $V(\mathbf{R})$ in which the zero spatial frequency and all spatial frequencies above twice the reciprocal-space probe-forming aperture cut-off are set to zero, to more fairly compare with the reconstructed phase which necessarily has these same restrictions (see Eq. (13)). Note that we have normalized out the nominally linear increase in phase with thickness to more meaningfully compare reconstruction fidelity of the scattering potential at different thicknesses. The second error metric is a percentage error of specific Fourier coefficients of the phase (or potential - as per Eq. (2) they are proportional within the ambit of the phase-object approximation). We will label the Fourier coefficients using Miller index notation: $f_{h k l}$.

Figs. 4(a) and (e) plot the MSE as a function of thickness for the $\mathrm{SrTiO}_{3}$ and $\mathrm{Al}_{3} \mathrm{Li}$ test samples, respectively. Data are shown for both the first-moment detector reconstruction, Eq. (13), and for a reconstruction based on the weak-phase-object approximation, Eq. (7). ${ }^{7}$ As expected, the plots show that the fidelity

\footnotetext{
${ }^{7}$ Since to explore the question of principle we are applying this analysis to perfect, simulated data, the analysis is accomplished by simply Fourier transforming the full 4D simulated STEM data over probe position to give $I\left(\mathbf{k}_{\perp}, \mathbf{K}\right)$, identifying the imaginary component $-\tilde{Q}_{i}$ and $\tilde{Q}_{r}$ are both real as both structures are centrosymmetric, and $T\left(\mathbf{k}_{\perp}\right)$ is real because the probe is assumed to be aberration-free - and dividing through by the transfer function. As the weak-
}

of the reconstructions break down with increasing thickness. The weak-phase-object approximation is seen to break down very quickly in the $\mathrm{SrTiO}_{3}$ case. In the $\mathrm{Al}_{3} \mathrm{Li}$ case, while the weak-phase-object approximation gives a poorer reconstruction fidelity than the first-moment detector reconstruction, the difference is smaller. For the strongly scattering $\mathrm{SrTiO}_{3}$ specimen, the first-moment detector reconstruction is seen to start breaking down beyond a mere $20 \AA$, whereas the weakly scattering $\mathrm{Al}_{3} \mathrm{Li}$ specimen appears good out towards $50 \AA$. To give some meaning to this metric, Figs. 4(b) and (f) show the reconstructions for the select thicknesses indicated by the boxes in Figs. 4(a) and (e), respectively. By $31 \AA$, the weak-phaseobject reconstruction in $\mathrm{SrTiO}_{3}$ is showing volcano-like structure on the $\mathrm{Sr}$ and $\mathrm{TiO}$ columns, whereas the first-moment detector reconstruction still bears good qualitative resemblance to the expected potential. By $55 \AA$, the first-moment detector reconstruction is also starting to qualitatively break down, showing volcano-like structure on the $\mathrm{Sr}$ and $\mathrm{TiO}$ columns, though the reconstruction of the phase of the pure $\mathrm{O}$ columns remains quite good. By contrast, there is little to visually distinguish the first-moment detector and weak-phase-object approximation reconstructions for the $\mathrm{Al}_{3} \mathrm{Li}$ case, though anomalous features appear in both reconstructions by a thickness of $127 \AA$. Figs. 4(c) and (g) show line scan extracts for the first-moment detector reconstructions for $\mathrm{SrTiO}_{3}$ and $\mathrm{Al}_{3} \mathrm{Li}$, respectively, and Figs. 4(d) and (h) show line scan extracts for the weak-phaseobject approximation reconstructions. Both the quantitative and qualitative differences between the two approximations are evident in these plots. This makes clear that the MSE in Fig. 4(e) is notably smaller than that in Fig. 4(a) not necessarily because the relative error is much smaller - comparison of Fig. $4(\mathrm{~g})$ with Fig. 4(c) shows the relative error to be broadly comparable - but rather because the potential for $\mathrm{Al}_{3} \mathrm{Li}$ is smaller than that for $\mathrm{SrTiO}_{3}$. It is also clear that the greatest error in the reconstruction occurs at the heavy element sites, $\mathrm{Sr}$ in $\mathrm{SrTiO}_{3}$ and, though less quickly, $\mathrm{Al}$ in $\mathrm{Al}_{3} \mathrm{Li}$. For later reference, note that reconstructions for thicker samples tend to underestimate the phase relative to what is expected from a perfect phase object. This is consistent with the mathematical similarity of the propagator with a diffusion problem [61], which acts to reduce sharp features in the wavefunction.

The percentage error of select Fourier coefficients of the phase for the $\mathrm{SrTiO}_{3}$ sample are plotted as a function of thickness in Fig. 5(a). It is seen that the percentage error in the high order Fourier coefficients generally grows faster than that in the lower order Fourier coefficients. As spatial propagation affects high spatial frequencies more strongly than low spatial frequencies, a reconstruction method that neglects spatial propagation might reasonably be expected to reconstruct high spatial frequencies less reliably than low spatial frequencies. Fig. 5(b) compares the percentage error for both a low-order Fourier coefficient, $f_{020}$, and a high-order Fourier coefficient, $f_{150}$, be-

phase-object approximation breaks down, different points $\mathbf{k}_{\perp}$ in the diffraction pattern may, via Eq. (7), predict different values for the same Fourier coefficient $\tilde{Q}_{i}(\mathbf{K})$. In the present reconstruction, all the different values determined from Eq. (7) were averaged to produce the "best estimate" for $\tilde{Q}_{i}(\mathbf{K})$. 

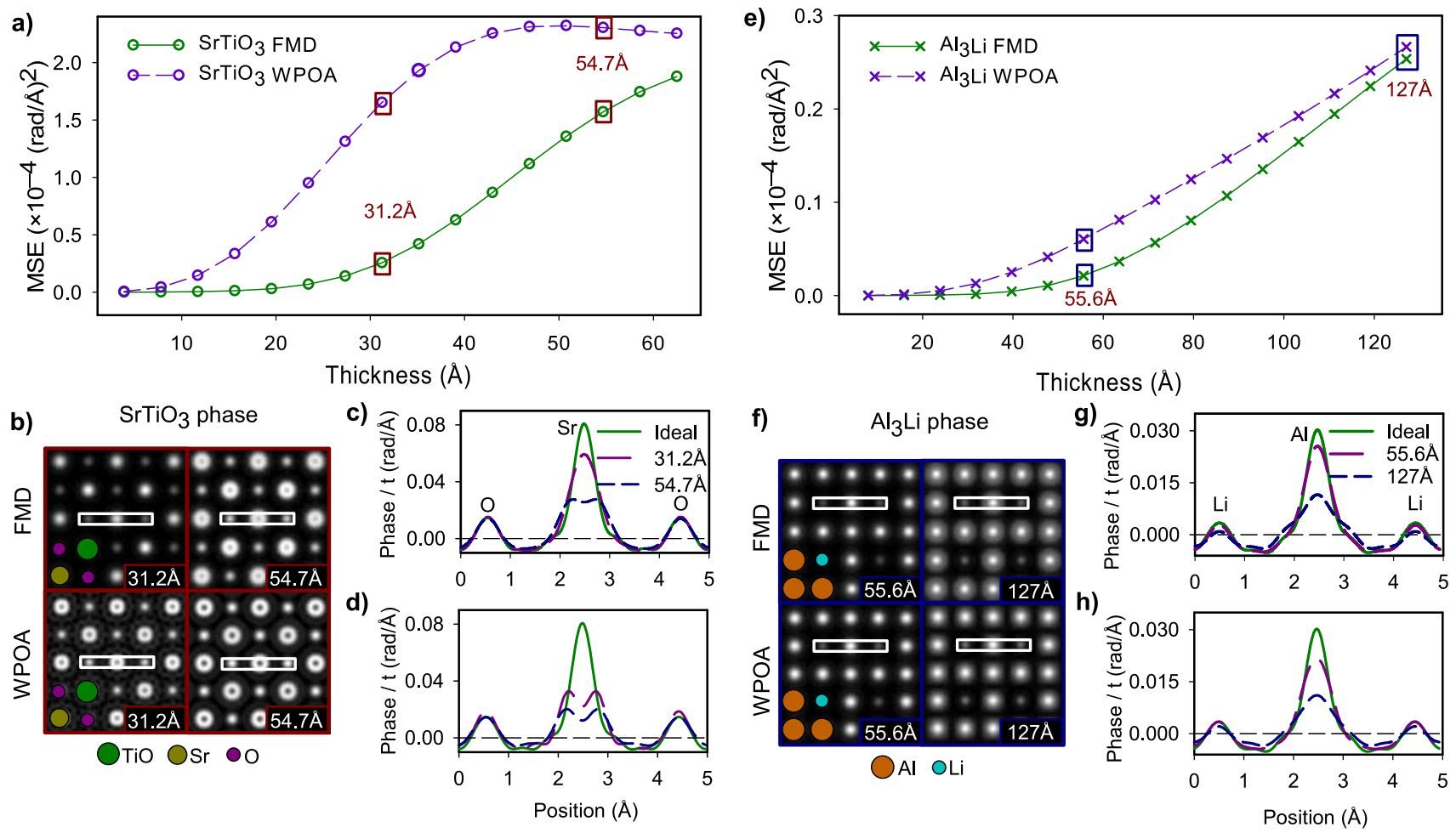

Figure 4: (a) Mean squared error (MSE) between the (bandwidth limited) ideal phase-object approximation phase and the reconstructed phase for $\mathrm{SrTiO}_{3}$, using both the first-moment detector (FMD) method, Eq. (13), and the weak-phase-object approximation (WPOA), Eq. (7). (b) Reconstructed potentials for the select thicknesses indicated by the boxes in (a). (c) FMD and (d) WPOA reconstruction line scan extracts from the white rectangles in (b). (e-h) As per (a-d) but for $\mathrm{Al}_{3} \mathrm{Li}$.

tween the first-moment detector reconstruction and the weakphase-object reconstruction. Consistent with Fig. 4, the weakphase-object reconstruction is poorer than the first-moment detector reconstruction, which is based on the more general phaseobject approximation.

\section{Approximating a first-moment detector using a seg- mented detector}

The previous section shows that the thickness range over which the phase-object approximation is valid for quantitative, atomic-resolution DPC imaging is quite restrictive: only a few tens of Angstrom for strongly scattering specimens ${ }^{8}$ and not much more for weakly scattering specimens. That was in the "best case scenario" of being able to synthesize a first-moment detector, i.e. evaluate Eq. (8). Proof-of-principle experiments collecting such 2D diffraction patterns as a function of probe scan position appear in the literature $[14,32]$. However, since these experiments are not yet routine, we explore the degree to which quantitative DPC might be approximated using the more established segmented detector geometry [9, 33, 34].

To approximate the first-moment detector expression of Eq. (8) using a combination of segmented detector signals in Eq.

\footnotetext{
${ }^{8}$ Note this restriction applies to quantitative analysis. As per the DPC tableau in Fig. 2, qualitatively interpretable DPC images for $\mathrm{SrTiO}_{3}$ persist to specimen thicknesses of at least $100 \AA$.
}

(5), we set

$$
D_{x, j}\left(\mathbf{k}_{\perp}\right)= \begin{cases}\left\{k_{x}\right\}_{\mathrm{CoM}, j} & \text { if } \mathbf{k}_{\perp} \text { lies within segment } j, \\ 0 & \text { otherwise }\end{cases}
$$

where $\left\{k_{x}\right\}_{\mathrm{CoM}, j}$ is the $x$-coordinate of the centre of mass of detector segment $j$ (an analytic expression is given in Appendix A for the case of an ideal segmented detector), and form

$$
\begin{aligned}
I_{x \text { seg. det. }}(\mathbf{R}) & =\sum_{j}\left[\int I\left(\mathbf{k}_{\perp}, \mathbf{R}\right) D_{x, j}\left(\mathbf{k}_{\perp}\right) d \mathbf{k}_{\perp}\right] \\
& \equiv \sum_{j}\left\{k_{x}\right\}_{\mathrm{CoM}, j} I_{j}(\mathbf{R}) .
\end{aligned}
$$

An analogous expression holds for the $y$ component. Together, these images can be used as input to the reconstruction expression of Eq. (13) under the assumption that they approximate the gradient of the phase imparted by the specimen.

We base our exploration on the 16-segment detector of Shibata et al. [34], which consists of four concentric, equal-width rings divided into quadrants. Fig. 6(a), in which the shaded area denotes the bright field disk, shows schematically a select number of promising configurations we shall consider, all of which are accessible by rotation of the detector and variation in the camera length. Case (i) uses just the four detector segments in the second annular ring - such that the inner angle is half the outer angle - with the bright field disk overlapping the 
a)

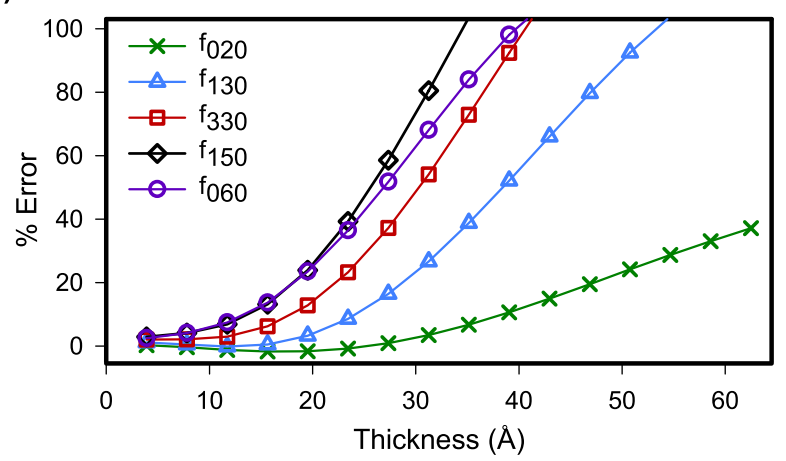

b)

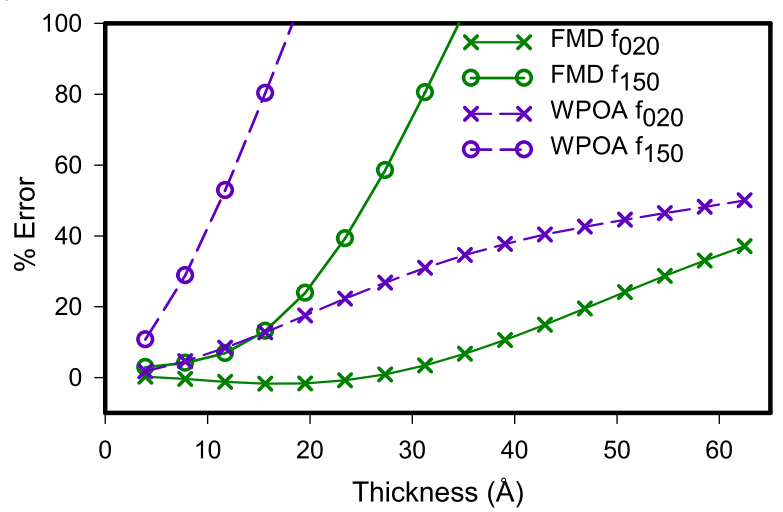

Figure 5: Comparison of the percentage error estimate in the reconstruction of (a) various Fourier coefficients in the first-moment detector (FMD) method and (b) select Fourier coefficients for both the FMD and the weak-phase-object approximation (WPOA) method for $\mathrm{SrTiO}_{3}$ as a function of thickness.

inner portion of the annulus. This is a common configuration for qualitative DPC imaging $[7,9,10]-$ it was used in generating the DPC image in Fig. 1(b) - and Chapman et al. [5] have shown that it favours low spatial frequency information, thereby helping to enhance contrast from long range fields relative to diffraction contrast in lower resolution imaging. In the idealization of rigid bright field disk displacement, Fig. 1(c), it would suffice. ${ }^{9}$ Since, as per Fig. 1(d), we expect a more complex diffraction pattern intensity redistribution, we might consider augmenting this with signals from the inner and outer rings, leading to configuration (ii) in Fig. 6(a) using 12 detector segments. Configuration (iii) has the same camera length as configuration (ii) but has the detector rotated by $45^{\circ}$. Configuration (iv) increases the camera length such that the bright field disk overlaps both inner rings of the detector, and also includes the outermost ring and thus 16 detector segments in total, slightly improving the sampling in the diffraction plane.

The percentage error for the Fourier coefficients $f_{020}$ and $f_{220}$ for a $\mathrm{SrTiO}_{3}$ specimen reconstructed using the detector configurations in Fig. 6(a) are shown in Fig. 6(b), with the first-moment detector results provided as reference. As per the

\footnotetext{
${ }^{9}$ Eq. (13) might not be the best reconstruction approach, but the segmented detector signals and disk deflection would be unambiguously related.
}
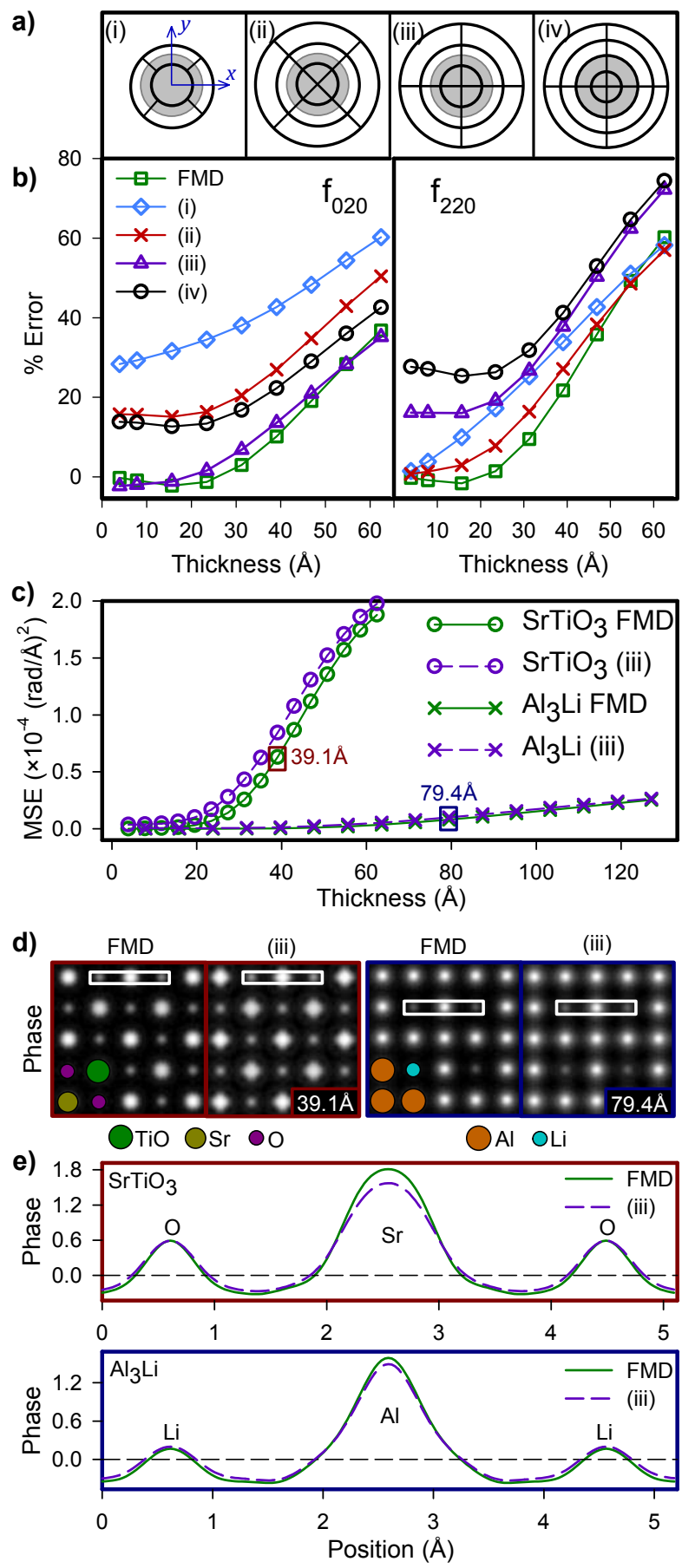

Figure 6: (a) Schematic of the detector configurations considered. The black lines denote the edges of the detector segments while the grey shading denotes the bright field region. (b) Percentage error of select Fourier coefficients as a function of thickness for the different detector configurations in (a). (c) MSE comparing the first-moment detector (FMD) with detector configuration (iii) from (a). (d) Reconstructed potentials for the select thicknesses denoted by boxes in (d). (e) Projected line scans from the white rectangles in (d).

previous section, all reconstructions get worse with increasing sample thickness. There is notable variation in reconstruction quality between the different detector configurations. For reconstructing the $f_{020}$ Fourier coefficient, configuration (iii) does 
almost as well as the first-moment detector, while the other configurations have poorer reconstruction fidelities. However, for reconstructing the $f_{220}$ Fourier coefficient, configuration (ii) gives the best agreement. None of the detector configurations is unilaterally better than the others across all frequencies, but, averaged over all Fourier coefficients, configurations (ii) and (iii) tend to be better than the others. Fig. 6(c) compares the MSE reconstruction fidelity measure between the first-moment detector and detector configuration (iii), with the latter giving an only marginally worse reconstruction according to this crude metric. The MSE for detector configuration (ii) is not shown, but is only marginally worse than that for detector configuration (iii) in this case. To give context to these numbers, Fig. 6(d) compares the two reconstructions for the two samples for the select thicknesses indicated by the boxes in Fig. 6(c). Projected line scans from these images are compared in Fig. 6(e). For $\mathrm{SrTiO}_{3}$, the detector configuration (iii) reconstruction gives the $\mathrm{Sr}$ peaks a square-ish appearance, further evidence of the variation in reconstruction fidelity between Fourier coefficients in different directions. Nevertheless, the line scans show quite favourable quantitative comparisons.

Fig. 6(e) shows the largest difference occurring on the strongly scattering columns, where the segmented detector underestimates the reconstructed phase relative to the firstmoment detector (which itself, as per Figs. 4(c) and (g), underestimates the reconstructed phase relative to the true result). The accuracy of the segmented detector reconstruction depends on how well the centre of mass of the detector segment approximates the centre of mass of the intensity distribution falling within that segment. In the absence of a sample, configuration (iv) in Fig. 6(a) would accurately reflect the centre of mass of the intensity distribution in each segment, whereas configurations (ii) and (iii) would overestimate the centre of mass of the intensity distribution in each segment in the second ring (the detector centre in ring 2 being $21.5 \mathrm{mrad}$ from the optical axis, whereas the intensity centre in ring 2 is only $17.5 \mathrm{mrad}$ ). However, with the specimen present, electron scattering tends to redistribute the intensity distribution to higher angles, with the result that, at least for these specimens, configurations (ii) and (iii) better estimate the first moment of the intensity distribution than does configuration (iv): despite its nominally better sampling of diffraction space, configuration (iv) tends to underestimate the first moment. It may be possible to find camera lengths which do better still. However, rather than seeking out maximally optimal conditions which likely depend on the sample and thickness, we emphasize that configuration (iii) has here proven to give good quantitative reconstructions for both the strongly scattering $\mathrm{SrTiO}_{3}$ sample and the weakly scattering $\mathrm{Al}_{3} \mathrm{Li}$ sample. Moreover, Fig. 6(e) compellingly suggests that evaluation of the first-moment using a pixel detector may give only a modest advance in quantitative accuracy over its estimate using segmented detectors for atomic resolution DPC imaging for such a configuration. (There is, of course, a lot more one can do with the intensity distribution on a full pixel detector than calculate the first moment, such as undertake a ptychographic analysis [51, 52], which might increase the resolution of the reconstruction, albeit at the expense of greater computational effort.)

\section{Experimental considerations and limitations}

Thus far our analysis has assumed somewhat idealized conditions. In this section we wish to assess the impact of several limitations of practice, specifically the effects of thermal scattering, non-optimum defocus, and shot noise.

The simulations presented thus far have included thermal scattering as an absorptive effect following Eq. (2), whereas the reconstruction has been performed assuming absorption to be negligible (i.e. following Eq. (11) rather than Eq. (9)). Simulations (not shown here) that do not include absorption in the multislice calculation give rise to a breakdown in reconstruction fidelity very similar to that in Fig. 4(a), suggesting there is little to be gained by attempting to correct for absorption within an absorptive model. What this model lacks, however, is the positive contribution to the diffraction intensity from electrons that have undergone thermal scattering. This can be included by performing simulations based on the frozen phonon model $[35,63]$.

Comparisons in reconstruction fidelity between simulations performed using the absorptive model and those using the frozen phonon model are shown in Fig. 7 for both a pixel (firstmoment) detector and detector configuration (iii) from Fig. 6(a). The fidelity of reconstruction in the frozen phonon simulations is found to be slightly better than the absorptive case. This is an example of compensating errors. Because the DPC signal is to some extent preserved in the distribution of thermally scattered electrons, the inclusion of the thermally scattered electrons increases the DPC signal and hence the reconstructed phase. This is especially true in the pixel detector case where the few electrons scattered to high angles are strongly weighted by the first-moment detector. This helps compensate for the underestimation that arises from analysing channelling data under the assumption of the phase-object approximation, improving the reconstruction fidelity. Consequently, though more by good fortune than by good management, the absorptive model fidelity analysis is if anything a little conservative, and we will therefore persist in using it.

Explorations thus far have assumed a probe defocus nominally (i.e. in the absence of scattering) putting the beam waist in the midplane of the crystal, since this has previously been established to be optimal for validity of the phase-object approximation [61, 62]. For very thin specimens, this is also likely to be the defocus condition that maximizes the HAADF signal [64] and therefore easy to determine experimentally. For thicker specimens, where admittedly Fig. 4 suggests we should not expect high resolution DPC imaging to be very reliable, the optimum defocus condition for HAADF tends to have the probe near to the entrance surface $[65,66]$. Fig. 8(a) shows how the reconstruction fidelity for select Fourier coefficients compares between the probe being focused on the specimen midplane and being focused on the specimen entrance surface. The latter is appreciably worse, especially for low order Fourier coefficients, for all but the thinnest of crystals. This makes it desirable for 


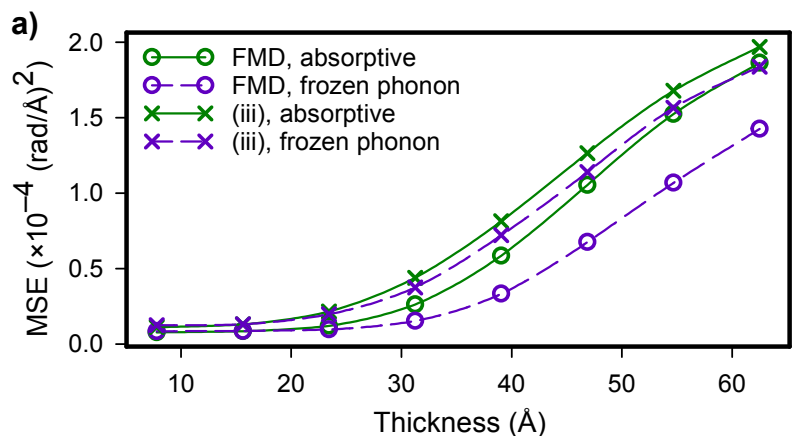

b)

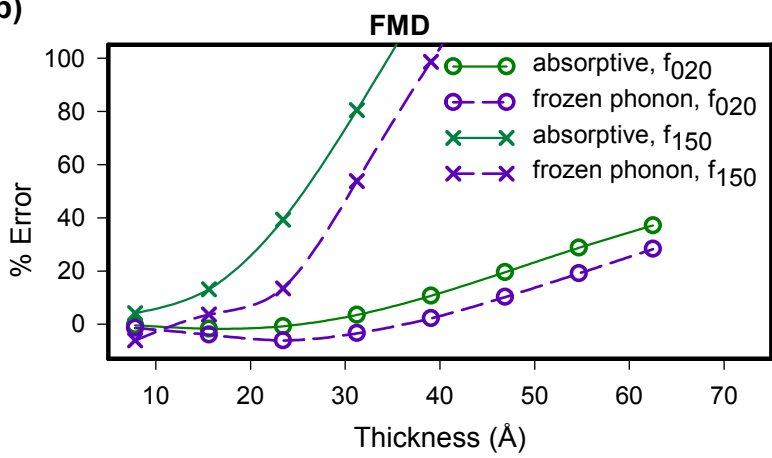

c)

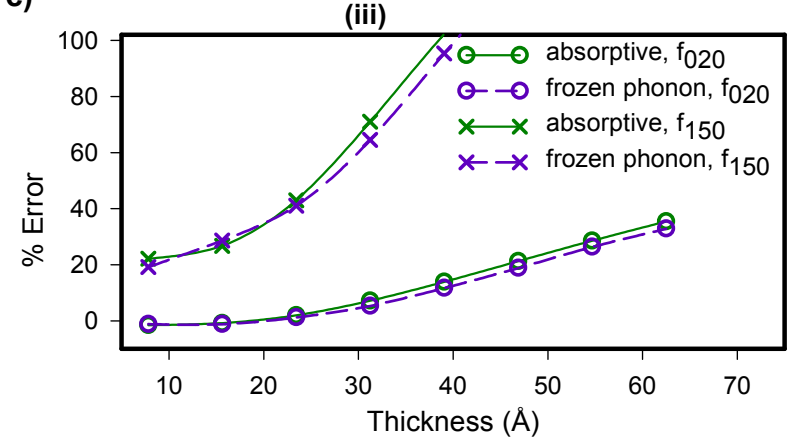

Figure 7: (a) MSE, and Fourier coefficient percentage errors for (b) the pixel (first-moment) detector and (c) detector approximation (iii) comparing the absorptive multislice method and the frozen phonon method. (Note: because of the "noise" introduced by slight under-convergence of the frozen phonon method, the MSE reconstructions here are based on a low-pass filtering slightly tighter than twice the reciprocal-space probe-forming aperture cut-off.)

DPC imaging to set the probe defocus to the specimen midplane.

Fig. 8(b) shows a simulated HAADF profile for a 31 A thick $\mathrm{SrTiO}_{3}$ specimen for a range of defocus values, taken from the mosaic of HAADF simulations in Fig. 8(c). The HAADF intensity on the TiO column peaks for a defocus of $-15.6 \AA$ (i.e. underfocus), which by geometric optics would put the beam waist in the specimen midplane. Fig. 8(d) shows that the signal averaged over a region between the columns (specifically, the blue hatched rectangle shown in Fig. 8(c)) also varies with defocus, reaching a minimum when the probe defocus nominally puts the beam waist at the specimen midplane. Figs. 8(e)-(g) show the same analysis for a $63 \AA$ thick $\mathrm{SrTiO}_{3}$ specimen. Now it is found that the defocus value at which the peak HAADF intensity occurs puts the nominal beam waist closer to the en- a)

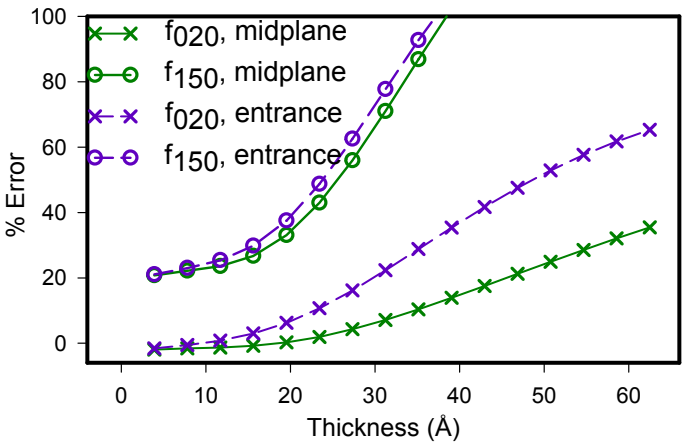

b)

$31.2 \AA$

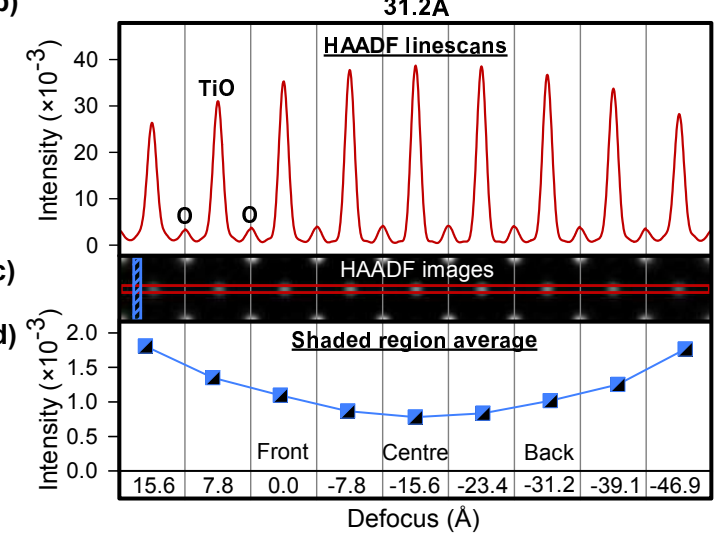

e)

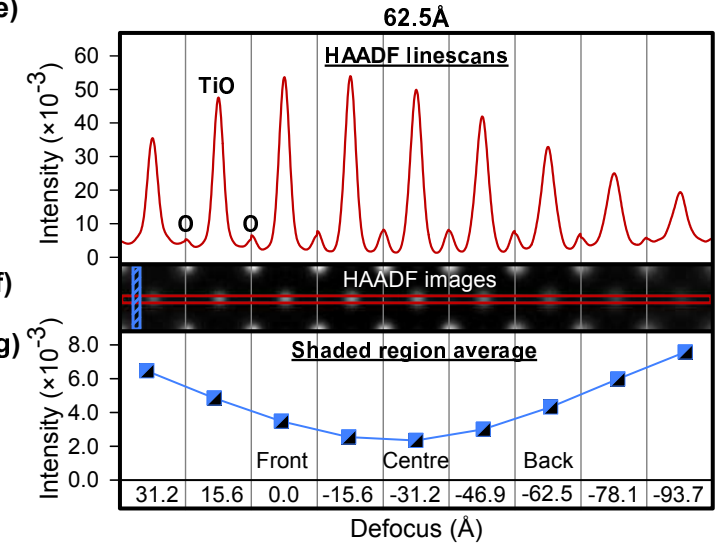

Figure 8: (a) Fourier coefficient percentage error plots as a function of thickness comparing the STEM probe being focused on the midplane of the sample with it being focused on the entrance surface, using detector configuration (iii) from Fig. 6(a). (b) Line extract through (c) the mosaic showing the $\mathrm{SrTiO}_{3} \mathrm{HAADF}$ images at various defocus values for a thickness of $31.2 \AA$. (d) Integrated signal from blue hatched rectangle in (c). (e-g) As per (b-d) but for a thickness of 62.5 $\AA$.

trance surface of the crystal [66, 67]. However, the average over the region between the columns again reaches its minimum for a probe defocus nominally putting the beam waist at the specimen midplane. This may offer a prescription for setting the defocus most favourably for DPC imaging. However, for the thinnest specimens, where quantitative DPC is expected to be most reliable, maximizing HAADF intensity should suffice.

The explorations thus far have been based on simulated data and are therefore noise-free. Müller et al. [14] have discussed the issue of scan noise - likely the greatest limitation in prac- 

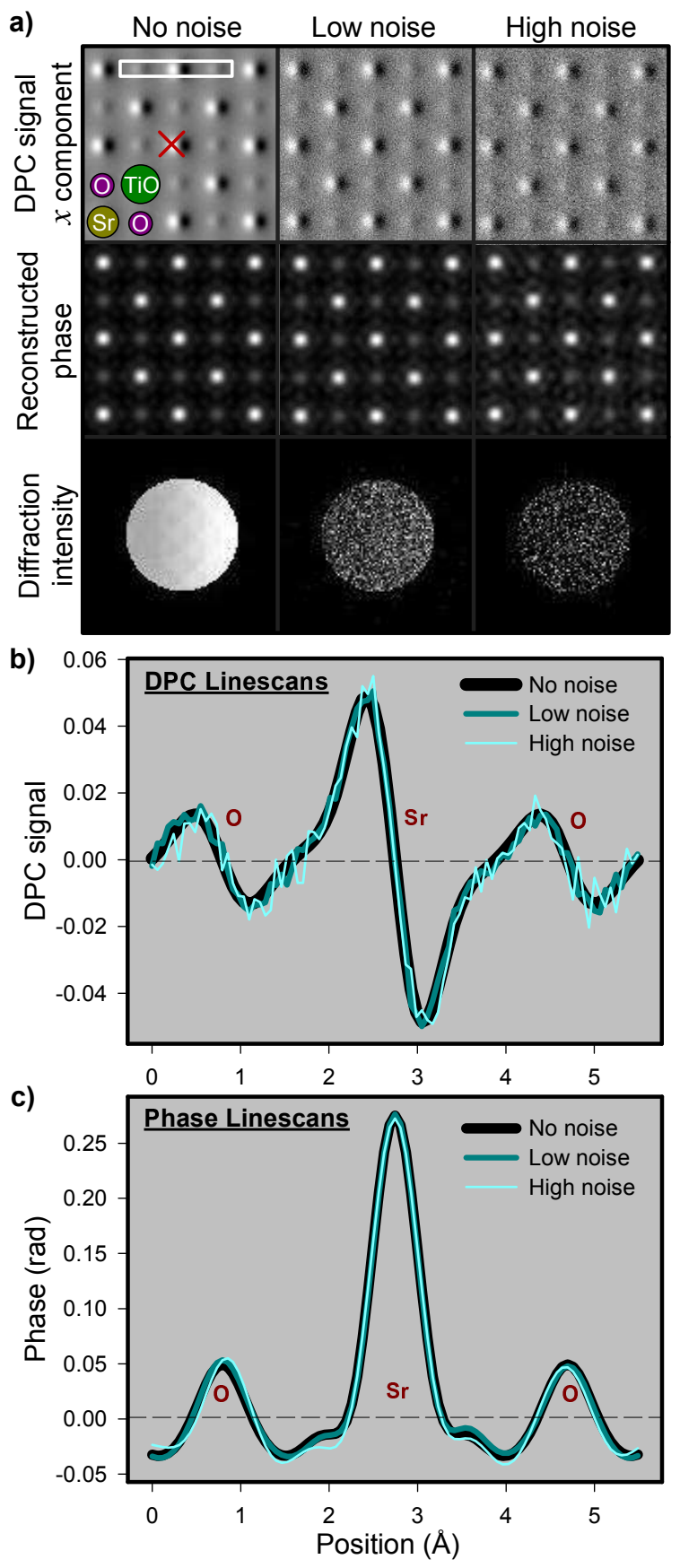

Figure 9: (a) Comparison of the DPC signal, reconstructed phase and select diffraction pattern for no noise, "low noise" and "high noise" conditions at the probe position marked with the red cross in the DPC image, assuming a single unit cell thickness of $\mathrm{SrTiO}_{3}$. Projected line scans corresponding to the white rectangle shown in (a) are given for the (b) DPC signal and (c) reconstructed phase.

tice. Here we consider the fundamental limitation of counting statistics. Instability and beam damage limit the amount of time the probe should be stationary at any given point, limiting the current in each recorded diffraction pattern and therefore meaning we should expect intensity fluctuations across the diffraction pattern from counting statistics. This is simulated in Fig. 9(a). The bottom row shows the diffraction pattern at one particular point with no noise and with what are identified as "low noise" and "high noise" conditions, corresponding to $1.5 \%$ and $3.0 \%$ shot noise on the number of counts in the full diffraction pattern per dwell point. The detriment to the visual appearance of the diffraction pattern is clear. However, as per Eq. (8), evaluating the DPC signal involves a (weighted) average over the diffraction pattern intensity. The top row in Fig. 9(a) shows the full simulated DPC images (for the $x$-direction) for these three cases, with line scan extracts shown in Fig. 9(b). The effect of noise on these DPC images is not nearly so dramatic as it is in the diffraction patterns. The reconstructions - middle row of Fig. 9(a) and extracted line scans in Fig. 9(c) - involve further integration and therefore a further smoothing effect as per Eq. (13). Noise nevertheless leads to reconstruction artefacts. A high pass filter removing spatial frequencies beyond $85 \%$ of the bandwidth limit of $\left|t\left(\mathbf{r}_{\perp}\right)\right|^{2}$ was applied to prevent the noise enhancement implied by small values of $\left|t\left(\mathbf{r}_{\perp}\right)\right|^{2}$ at high frequencies in Eq. (13). A low pass filter removing spatial frequencies below $5 \%$ of the bandwidth limit of $\left|t\left(\mathbf{r}_{\perp}\right)\right|^{2}$ was applied to remove the noise enhancement implied by small values of $\left|k_{x}+i k_{y}\right|$ at low frequencies in Eq. (13). The comparisons in Figs. 9(a) and (c) show that, post-filtering, the reconstruction is robust in the presence of shot noise.

It should be noted that we have considered shot noise on an ideal, background-free signal. In practice, signal indistinguishable from background noise, for instance in the detection electronics, will not allow for DPC reconstruction. Nevertheless, on the question of principle, the reconstruction procedure is robust with respect to shot noise.

\section{Qualitative phase reconstruction from experimental data}

In the proof-of-principle demonstration of atomic resolution DPC imaging using a segmented detector by Shibata et al. [10], experimental DPC images were compared against simulated DPC images both using detector configuration (i) from Fig. 6(a). That sufficed to show that the form of the DPC images was consistent with expectations for those imaging conditions. To round out the present discussion, we reprocess that data to test the application of Eq. (13) to experimental data. Though quantitative comparison of STEM intensities between experiment and simulation is achievable (e.g. Ref. [68]), insufficient data were collected in the Shibata et al. DPC imaging experiment [10] to set an absolute intensity scale, and as a consequence the phase cannot be reconstructed quantitatively. Nevertheless, we can examine the qualitative reconstruction fidelity of applying Eq. (13) to real experimental data.

Fig. 10(a) shows a section of the experimental HAADF image from $\mathrm{SrTiO}_{3}$ viewed along the [001] zone axis, which serves as our reference for identifying the location of the $\mathrm{Sr}$, $\mathrm{TiO}$ and $\mathrm{O}$ columns (the last are not directly visible, but their location follows from the known structure). Figs. 10(b) and (c) show the DPC images for the $x$ and $y$ directions formed using Eq. (16) and a detector configuration oriented as per Fig. 

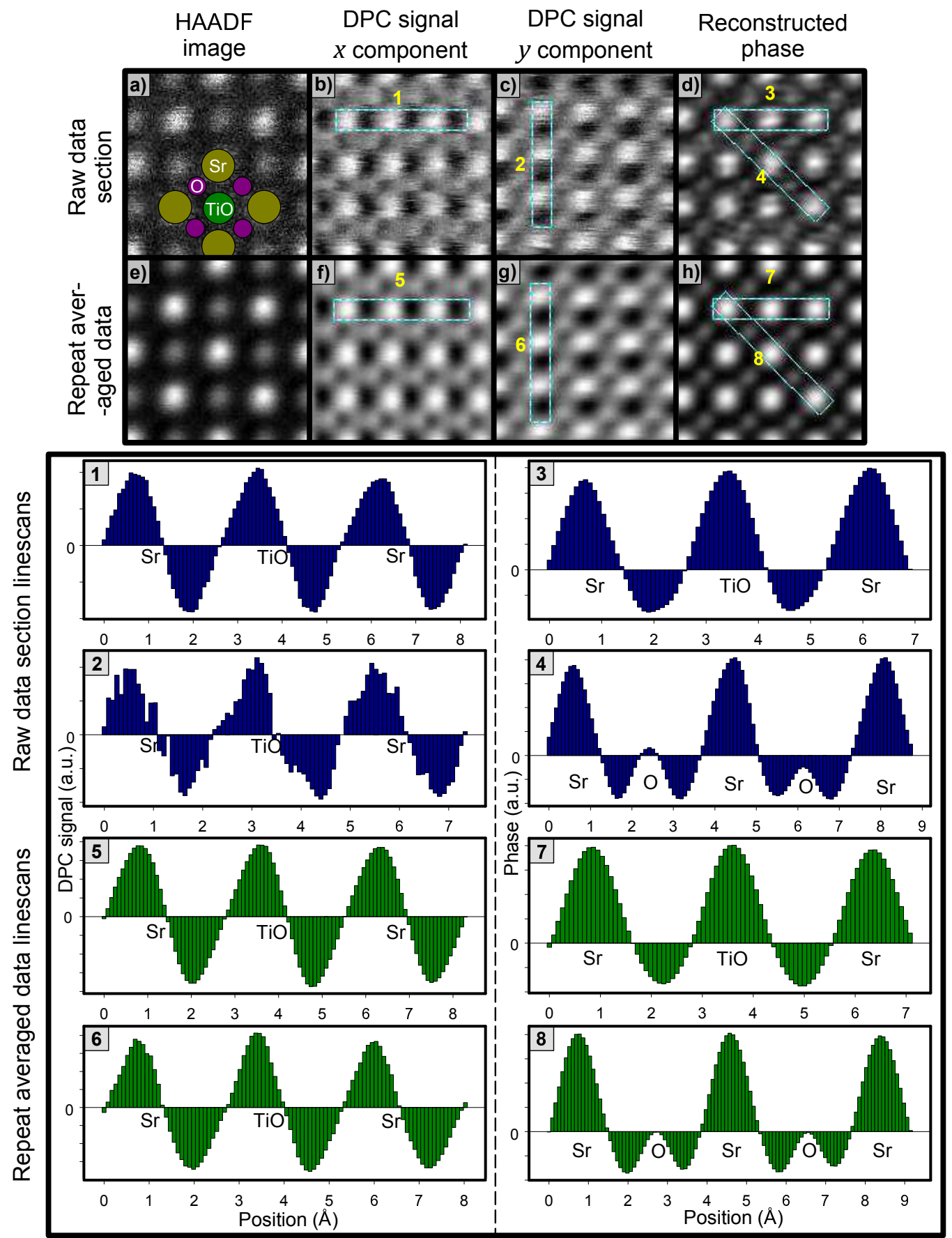

Figure 10: Raw and repeat-unit-averaged HAADF, DPC and reconstructed phase images (using detector configuration (ii)) images of SrTiO 3 for a $\sim 30 \AA$ thick specimen oriented along the [001] zone axis (adapted from data in Ref. [10]). The projected line scans in the lower portion of the figure are taken from the corresponding numbered rectangles in the images in the upper portion. In the reconstructions presented, both low-pass and high-pass filters have been applied. 
6(a) (ii), ${ }^{10}$ i.e. using 12 segments rather than the 4 used in Fig. 1(b), even though qualitatively these images look very similar. Scan noise is evident in all these images. In particular, line scan extracts \#1 and \#2 show that there is some column-to-column variation in peak height and shape between nominally equivalent columns. Because a larger field of view was recorded, we can endeavour to reduce these effects by repeat-unit averaging. Results for the repeat-unit averaged HAADF and DPC images are shown in Figs. 10(e)-(g) and line scan extracts \#5 and \#6, which show much better uniformity.

Reconstructions using Eq. (13) for the raw and repeat-unit averaged data sets are shown in Figs. 10(d) and (h) and the corresponding line scan extracts \#3, \#4, \#7 and \#8. Consistent with the experimental conditions, the reconstruction assumed $200 \mathrm{keV}$ electrons and a $23 \mathrm{mrad}$ probe-forming aperture angle. The reconstruction assumed the defocus was set to the specimen midlane, which is probably not exactly true but likely a good approximation: Ref. [10] estimated a specimen thickness of $31 \AA$ and a defocus of $-11 \AA$. Moreover, spatial incoherence (also referred to as finite effective source size) has not been accounted for in our reconstruction since it was not characterized in the experiment, though its presence is evident in the difference in peak width between Figs. 10(d) and (h) and those in Fig. 4(b). As per the discussion on noise in section 6, both high-pass (spatial frequencies above $1.56 \AA^{-1}$, i.e. $85 \%$ of the twice the probe-forming aperture cut-off resolution limit) and low-pass (below $0.092 \AA^{-1}$, i.e. $5 \%$ of the twice the probe-forming aperture cut-off resolution limit) filters have been applied to regularize against the effects of noise and scan distortion.

The qualitative fidelity of the reconstruction seems reasonable. The $\mathrm{O}$ columns are clearly visible, though notably fainter than both the $\mathrm{Sr}$ and $\mathrm{TiO}$ columns which are of comparable height to one another. This is consistent with the $\mathrm{SrTiO}_{3}$ simulations in Figs. 4(b) and (c). The reconstructions look smoother than the input data since phase reconstruction from DPC signals inherently involves the smoothing operation of integration and we have further applied regularization via filtering, though some of the variation in peak height between nominally equivalent columns persists. An assessment of quantitative reconstruction fidelity must wait until a sufficiently characterized experiment is performed that allows it, but Fig. 10 suggests no inprinciple problems in performing such reconstructions on experimental data from a segmented detector.

\section{Conclusion}

For quantitative phase reconstruction from atomic-resolution DPC STEM images, the idealized first-moment detector, realizable using pixel detectors, can be tolerably approximated via a segmented detector according to the metric of mean square

\footnotetext{
${ }^{10} \mathrm{We}$ earlier advocated that configuration (iii) gave on average a slightly better fidelity than configuration (ii) for the reconstruction process applied to $\mathrm{SrTiO}_{3}$. The analysis of Fig. 10 assumes configuration (ii) because it is a reprocessing of data from Ref. [10] which was collected using the detector orientation of configuration (ii). As the average difference between configurations (ii) and (iii) is very slight, this is not deemed to be a significant limitation.
}

error in the reconstructed potentials, though the fidelity with which specific Fourier coefficients of phase/potential are reconstructed varies appreciably. The reconstructions are fairly sensitive to defocus, though quite robust to noise and thermal scattering. The major limitation to first-moment DPC analysis for both pixel detector and segmented detector phase reconstructions is the highly restrictive validity domain of the phase-object approximation, which is of the order of $50 \AA$ for very weakly scattering specimens like $\mathrm{Al}_{3} \mathrm{Li}$ and even smaller for more strongly scattering specimens like $\mathrm{SrTiO}_{3}$. It appears that simulations must remain an essential adjunct to atomic resolution DPC STEM image interpretation for all but the very thinnest of specimens.

\section{Acknowledgements}

S.D.F. thanks M.D. de Jonge and L.J. Allen for helpful discussions. This research was supported under the Australian Research Councils Discovery Projects funding scheme (Projects DP110101570 and DP140102538). N.S. acknowledges support from SENTAN, JST and JSPS KAKENHI Grant number 26289234.

\section{Appendix A. Segment "centre of mass"}

Consider a single detector segment as shown schematically in Fig. A.11. We define a polar coordinate system centred on the optical axis where the radial distance is denoted by $k$ (the reciprocal space distance associated with a given scattering angle $\beta$ via $k=\tan (\beta) / \lambda)$ and the polar angle is denoted by $\theta$. The zero polar angle is chosen to bisect the detector segment. (Results with respect to other orientations follow from the present case by trivial trigonometric projection.) By symmetry, the "centre of mass" of the detector segment will lie along this line. It remains to find its radial location, $k_{\mathrm{CoM}}$ :

$$
k_{\mathrm{CoM}} \equiv \frac{\int_{-\theta_{1}}^{\theta_{1}} \int_{k_{1}}^{k_{2}} k \cos (\theta) k d k d \theta}{\int_{-\theta_{1}}^{\theta_{1}} \int_{k_{1}}^{k_{2}} k d k d \theta}=\frac{2}{3} \frac{\sin \left(\theta_{1}\right)}{\theta_{1}} \frac{k_{2}^{3}-k_{1}^{3}}{k_{2}^{2}-k_{1}^{2}} .
$$

For the quadrant detector, $\theta_{1}=\pi / 4$ and so

$$
k_{\mathrm{CoM}}=\frac{4 \sqrt{2}}{3 \pi} \frac{k_{2}^{3}-k_{1}^{3}}{k_{2}^{2}-k_{1}^{2}}
$$

\section{References}

\section{References}

[1] H. Rose, Phase contrast in scanning transmission electron microscopy, Optik 39 (1974) 416-436.

[2] H. Rose, Nonstandard imaging methods in electron microscopy, Ultramicroscopy 2 (1977) 251-267.

[3] N. H. Dekkers, H. de Lang, Differential phase contrast in a STEM, Optik 41 (1974) 452-456.

[4] J. N. Chapman, P. E. Batson, E. M. Waddell, R. P. Ferrier, The direct determination of magnetic domain wall profiles by differential phase contrast electron microscopy, Ultramicroscopy 3 (1978) 203-214. 


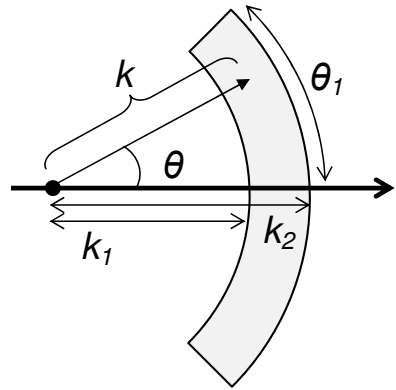

Figure A.11: Schematic of the polar coordinate system used to determine the "centre of mass" of the detector segment.

[5] J. N. Chapman, I. McFadyen, S. McVitie, Modified differential phase contrast lorentz microscopy for improved imaging of magnetic structures, IEEE Trans. Magnetics 26 (1990) 1506-1511.

[6] J. N. Chapman, R. Ploessl, D. M. Donnet, Differential phase contrast microscopy of magnetic materials, Ultramicroscopy 47 (1992) 331-338.

[7] T. Uhlig, J. Zweck, Direct observation of switching processes in permalloy rings with lorentz microscopy, Phys. Rev. Lett. 93 (2004) 047203.

[8] T. Uhlig, M. Rahm, C. Dietrich, R. Hölinger, M. Heumann, D. Weiss, J. Zweck, Shifting and pinning of a magnetic vortex core in a permalloy dot by a magnetic field, Phys. Rev. Lett. 95 (2005) 237205.

[9] M. Lohr, R. Schregle, M. Jetter, C. Wächter, T. Wunderer, F. Scholz, J. Zweck, Differential phase contrast 2.0-opening new "fields" for an established technique, Ultramicroscopy 117 (2012) 7-14.

[10] N. Shibata, S. D. Findlay, Y. Kohno, H. Sawada, Y. Kondo, Y. Ikuhara, Differential phase-contrast microscopy at atomic resolution, Nature Physics 8 (2012) 611-615.

[11] B. Bauer, J. Hubmann, M. Lohr, E. Reiger, D. Bougeard, J. Zweck, Direct detection of spontaneous polarization in wurtzite GaAs nanowires, Applied Physics Letters 104 (2014) 211902.

[12] N. Shibata, S. D. Findlay, H. Sasaki, T. Matsumoto, H. Sawada, Y. Kohno, S. Otomo, R. Minato, Y. Ikuhara, Imaging of built-in electric field at a p-n junction by scanning transmission electron microscopy, Scientific Reports 5 (2015) 10040.

[13] A. Lubk, J. Zweck, Differential phase contrast: an integral perspective, Phys. Rev. A 91 (2015) 023805.

[14] K. Müller, F. F. Krause, A. Béché, M. Schowalter, V. Galioit, S. Löffler, J. Verbeeck, J. Zweck, P. Schattschneider, A. Rosenauer, Atomic electric fields revealed by quantum mechanical approach to electron picodiffraction, Nature Communications 5 (2014) 5653.

[15] S. J. Pennycook, D. E. Jesson, High-resolution Z-contrast imaging of crystals, Ultramicroscopy 37 (1991) 14-38.

[16] G. R. Morrison, J. N. Chapman, A comparison of three differential phase contrast systems suitable for use in STEM, Optik 64 (1983) 1-12.

[17] S. Majert, H. Kohl, High-resolution STEM imaging with a quadrant detector - Conditions for differential phase contrast microscopy in the weak phase object approximation, Ultramicroscopy 148 (2014) 81.

[18] R. E. Burge, P. van Toorn, Multi-signal detection and processing in STEM, Inst. Phys. Conf. Ser. 52 (1980) 249-252.

[19] M. N. Landauer, B. McCallum, J. Rodenburg, Double resolution imaging of weak phase specimens with quadrant detectors in STEM, Optik 100 (1995) 37-46.

[20] B. C. McCallum, M. N. Landauer, J. M. Rodenburg, Complex image reconstruction of weak specimens from a three-sector detector in STEM, Optik 101 (1995) 53-62.

[21] M. Hammel, H. Rose, Optimum rotationally symmetric detector configurations for phase contrast imaging in scanning transmission electron microscopy, Ultramicroscopy 58 (1995) 403-415.

[22] T. Ishida, T. Kawasaki, T. Tanji, T. Kodama, T. Matsutani, K. Ogai, T. Ikuta, Phase reconstruction in annular bright-field scanning transmission electron microscopy, Microscopy, in press, doi:10.1093/jmicro/dfu098.

[23] T. J. Pennycook, A. R. Lupini, H. Yang, M. F. Murfitt, L. Jones, P. D. Nellist, Efficient phase contrast imaging in STEM using a pixelated de- tector. Part 1: Experimental demonstration at atomic resolution, Ultramicroscopy 151 (2015) 160.

[24] H. Yang, T. J. Pennycook, P. D. Nellist, Efficient phase contrast imaging in STEM using a pixelated detector. Part II: Optimisation of imaging conditions, Ultramicroscopy 151 (2015) 350.

[25] D. F. Lynch, A. F. Moodie, M. A. O'Keefe, n-Beam lattive images. V. The use of the change-density approximation in the interpreation of lattice images, Acta Cryst. A31 (1975) 300-307.

[26] M. Vulović, L. M. Voortman, L. J. van Vliet, B. Rieger, When to use the projection assumption and the weak-phase object approximation in phase contrast cryo-EM, Ultramicroscopy 136 (2014) 61-66.

[27] D. L. Misell, On the validity of the weak-phase and other approximations in the analysis of electron microscope images, J. Phys. D 9 (1976) 18491866.

[28] F. H. Li, D. Tang, Pseudo-weak-phase-object approximation in highresolution electron microscopy. I. Theory, Acta Cryst. A41 (1985) 376382.

[29] T. Ishida, T. Kawasaki, T. Tanji, T. Ikuta, Quantitative evaluation of annular bright-field phase images in STEM, Microscopy, accepted.

[30] E. M. Waddell, J. N. Chapman, Linear imaging of strong phase objects using asymmetrical detectors in STEM, Optik 54 (1979) 83-96.

[31] M. D. de Jonge, B. Hornberger, C. Holzner, D. Legnini, D. Patterson, I. McNulty, C. Jacobsen, S. Vogt, Quantitative phase imaging with a scanning transmission X-ray microscope, Phys. Rev. Lett. 100 (2008) 163902.

[32] K. Kimoto, K. Ishizuka, Spatially resolved diffractometry with atomiccolumn resoltuion, Ultramicroscopy 111 (2011) 1111-1116.

[33] M. Haider, A. Epstein, P. Jarron, C. Boulin, A versatile, software configurable multichannel STEM detector for angle-resolved imaging, Ultramicroscopy 54 (1994) 41-59.

[34] N. Shibata, Y. Kohno, S. Findlay, H. Sawada, Y. Kondo, Y. Ikuhara, New area detector for atomic-resolution scanning transmission electron microscopy, J. Electron Microscopy 59 (2010) 473-479.

[35] E. J. Kirkland, Advanced computing in electron microscopy, Plenum Press, New York and London, 1998.

[36] W. Coene, G. Janssen, M. O. de Beeck, D. Van Dyck, Phase retrieval through focus variation for ultra-resolution in field-emission transmission electron microscopy, Phys. Rev. Lett. 69 (1992) 3743-3746.

[37] W. M. J. Coene, A. Thust, M. Op de Beeck, D. Van Dyck, Maximumlikelihood method for focus-variation image reconstruction in high resolution transmission electron microscopy, Ultramicroscopy 64 (1996) 109_ 135 .

[38] C. Jia, A. Thust, Investigation of atomic displacements at a $\Sigma 3\{111\}$ twin boundary in $\mathrm{BaTiO}_{3}$ by means of phase-retrieval electron microscopy, Phys. Rev. Lett. 82 (1999) 5052-5055.

[39] L. J. Allen, M. P. Oxley, Phase retrieval from series of images obtained by defocus variation, Opt. Comm. 199 (2001) 65-75.

[40] R. R. Meyer, A. I. Kirkland, W. O. Saxton, A new method for the determination of the wave aberration function for high resolution TEM - 1: measurement of the symmetric aberrations, Ultramicroscopy 92 (2002) 89-109.

[41] L. J. Allen, W. McBride, N. L. O'Leary, M. P. Oxley, Exit wave reconstruction at atomic resolution, Ultramicroscopy 100 (2004) 91-104.

[42] A. Kikland, W. Saxton, K.-L. Chau, K. Tsuno, M. Kawasaki, Superresolution by aperture synthesis: tilt series reconstruction in ctem, Ultramicrsocopy 57 (1995) 355.

[43] S. Haigh, H. Sawada, A. Kikland, Atomic structure imaging beyond the conventional resolution limits in the transmission electron microscope, Phys. Rev. Lett. 103 (2009) 126101.

[44] J. Zuo, I. Vartanyants, M. Gao, R. Zhang, L. Nagahara, Atomic resolution imaging of a carbon nanotube from diffraction intensities, Science 300 (2003) 1419.

[45] J. Wu, U. Weierstall, J. Spence, Diffractive electron imaging of nanoparticles on a substrate, Nature Materials 4 (2005) 912.

[46] S. Morishita, J. Yamasaki, K. Nakamura, T. Kato, N. Tanaka, Diffractive imaging of the dumbbell structure in silicon by spherical-aberrationcorrected electron diffrction, Appl. Phys. Lett. 93 (2008) 183103.

[47] W. Huang, J. Zuo, B. Jiang, K. Kwon, M. Shim, Sub-ångström-resolution diffractive imaging of single nanocrystals, Nature Materials 4 (2005) 912.

[48] H. Lichte, M. Lehmann, Electron holography - basics and applications, Rep. Prog. Phys. 71 (2008) 016102.

[49] M. Lick, B. Freitag, S. Kujawa, M. Lehmann, T. Niermann, State of the art 
in atomic resolution off-axis electron holography, Ultramicroscopy 116 (2012) 13-23.

[50] J. M. Rodenburg, R. H. T. Bates, The theory of super-resolution electron microscopy via Wigner-distribution deconvolution, Phil. Trans. R. Soc. Lond. A 339 (1992) 521-553.

[51] H. M. L. Faulkner, J. M. Rodenburg, Movable aperture lensless transmission microscopy: a novel phase retrieval algorithm, Phys. Rev. Lett. 93 (2004) 023903.

[52] J. M. Rodenburg, H. M. L. Faulkner, A phase retrieval algorithm for shifting illumination, Appl. Phys. Lett. 85 (2004) 4795-4797.

[53] M. Humphry, B. Kraus, A. Hurst, A. Maiden, J. Rodenburg, Ptychographic electron microscopy using high-angle dark-field scattering for sub-nanometre resolution imaging, Nature Communications 3 (2012) 730.

[54] C. T. Putkunz, A. J. D’Alfonso, A. J. Morgan, M. Weyland, C. Dwyer, L. Bourgeois, J. Etheridge, A. Roberts, R. E. Scholten, K. A. Nugent, L. J. Allen, Atom-scale ptychographic electron diffractive imaging of boron nitride cones, Phys. Rev. Lett. 108 (2012) 073901.

[55] A. Morgan, A. D'Alfonso, P. Wang, H. Sawada, A. Kirkland, L. Allen, Fast deterministic single-exposure coherent diffractive imaging at subÅngström resolution, Phys. Rev. B 87 (2013) 094115.

[56] A. J. D'Alfonso, A. J. Morgan, A. W. C. Yan, P. Wang, H. Sawada, A. I. Kirkland, L. J. Allen, Deterministic electron ptychography at atomic resolution, Phys. Rev. A 89 (2014) 064101.

[57] P. Nellist, B. McCallum, J. Rodenburg, Resolution beyond the 'information limit' in transmission electron microscopy, Nature 374 (1995) 630632.

[58] A. M. Maiden, M. J. Humphry, J. M. Rodenburg, Ptychographic transmission microscopy in three dimensions using a multi-slice approach, J. Opt. Soc. Am. A 29 (2012) 1606-1614.

[59] J. M. Cowley, Configured detectors for STEM imaging of thin specimens, Ultramicroscopy 49 (1993) 4-13.

[60] M. Piana, M. Bertero, Regularized deconvolution of multiple images of the same object, J. Opt. Soc. Am. A 13 (1996) 1516-1523.

[61] D. Van Dyck, Image calculation in high-resolution electron microscopy: problems, progress, and prospects, Adv. Electron. Electron Phys. 65 (1985) 295-355

[62] T. Plamann, J. M. Rodenburg, Electron ptychography. II. Theory of threedimensional propagation effects, Acta Cryst. A54 (1996) 61-73.

[63] R. F. Loane, P. Xu, J. Silcox, Thermal vibrations in convergent-beam electron diffraction, Acta Cryst. A47 (1991) 267-278.

[64] H. L. Xin, D. A. Muller, Three-dimensional imaging in aberrationcorrected electron microscopes, Microscopy and Microanalysis 16 (2010) 445-455.

[65] Y. Peng, P. D. Nellist, S. J. Pennycook, HAADF-STEM imaging with sub-angstrom probes: a full Bloch wave analysis, J. Electron Microscopy 53 (2004) 257-266.

[66] A. J. D'Alfonso, S. D. Findlay, M. P. Oxley, S. J. Pennycook, K. van Benthem, L. J. Allen, Depth sectioning in scanning transmission electron microcopy based on core-loss spectroscopy, Ultramicroscopy 108 (2007) $17-28$.

[67] E. C. Cosgriff, P. D. Nellist, A Bloch wave analysis of optical sectioning in aberration-corrected STEM, Ultramicroscopy 107 (2007) 626-634.

[68] J. M. LeBeau, S. D. Findlay, L. J. Allen, S. Stemmer, Quantitative atomic resolution scanning transmission electron microscopy, Phys. Rev. Lett. 100 (2008) 206101. 